\begin{tabular}{|l|l|l|l|}
\hline Eiszeitalter $u$. Gegenwart & 32 & $\begin{array}{c}81-91 \\
4 \mathrm{Abb} .5 \mathrm{Tab} .\end{array}$ & Hannover 1982 \\
\hline
\end{tabular}

\title{
Die Bedeutung des Feinheitsgrades als geomorphologische Auswertungsmethode
}

\author{
Helmut Siebertz *)
}

Method, geomorphology, map, interpretation, classification, fineness, loess, quicksand, wind sedimentation, sampling, size distribution, paleontological reconstruction, paleography. Lower Rhine Lowlands, Nordrhein-Westfalen, TK 25. Nr.: 4203, 4303

Kurzfassung: Der Feinheitsgrad ist eine Kennzahl, die eine Aussage über die Zusammensetzung einer Sedimentprobe gibt und zum schnelleren Vergleich von Proben untereinander dient.

Mit Hilfe des Feinheitsgrades können Sedimente klassifiziert (Erstellung von Sedimentnomenklaturen) und Sedimentationsräume gegliedert werden. Ferner kann man mit dem Feinheitsgrad die Rekonstruktion paläogeographischer Windverhältnisse vornehmen und die Bodenart bestimmen.

\section{[The Meaning of the Degree of Fineness as Geomorphological Measurement]}

A bstract: The degree of fineness is an index of the composition of sedimentary samples and makes it easear to compare different samples to each other.

It also helps (1) to classify sedimentary deposits (to set up sediment nomenclatures), (2) to distinguish between areas of different sediments, (3) to reconstruct paleogeographical wind directions and (4) to define the soil class.

\section{Einleitung}

Das Ergebnis granulometrischer Laboranalysen von Boden- oder Sedimentproben ist häufig recht differenziert. Dies ist u. a. von der Korngrößenklassifikation abhängig, die der Untersuchung zugrundegelegt wird. Je nach Fragestellung kann für die Auswertung eine mehr oder weniger differenzierte Einteilung zugrundegelegt werden.

Eine gebräuchliche und häufig benutzte Korngrößenklassifikation in der Bundesrepublik Deutschland ist z. B. die aus neun Kornfraktionen bestehende logarithmische Einteilung nach DIN 4188 (vgl. KösTER 1964).

Nach SchöNhals (1955) genügt für viele Untersuchungen die Ermittlung der Korngrößenzusammensetzung und ihre graphische Darstellung (s. Tab. 1-3), wobei die Vergleichbarkeit von Kornanalysendaten von großer Bedeutung ist. Voraussetzung allerdings ist, daß eine Einheitlichkeit in den Untersuchungs- und Auswertungsmethoden vorliegt, um von der Bearbeitungsmethode her Ungleichheiten und Fehlerquellen zu vermeiden.

Um eine Sedimentprobe als Ganzes zu charakterisieren, muß ein Wert geschaffen werden, der als Repräsentant für die jeweilige Probe in Erscheinung tritt und je nach unterschiedlicher Sedimentzusammensetzung charakteristische Werte liefert, die miteinander in Beziehung gesetzt werden können. Da der Aussagewert einer einzelnen Sedimentprobe kaum von Bedeutung ist und für die Aussagekraft der Landschaftsgenese Reihenuntersuchungen durchgeführt werden müssen (LESER 1966), ist eine Standardisierung der einzelnen Korngrößenanalysen durch einen Repräsentativwert wichtig und arbeitstechnisch bedeutsam (vgl. SieberTz 1980).

*) Anschrift des Autors: Dipl.-Geogr. Dr. H. S i e b e r t z, Seminar für Geographie, Pädagogische Fakultät, Universität Bonn. 

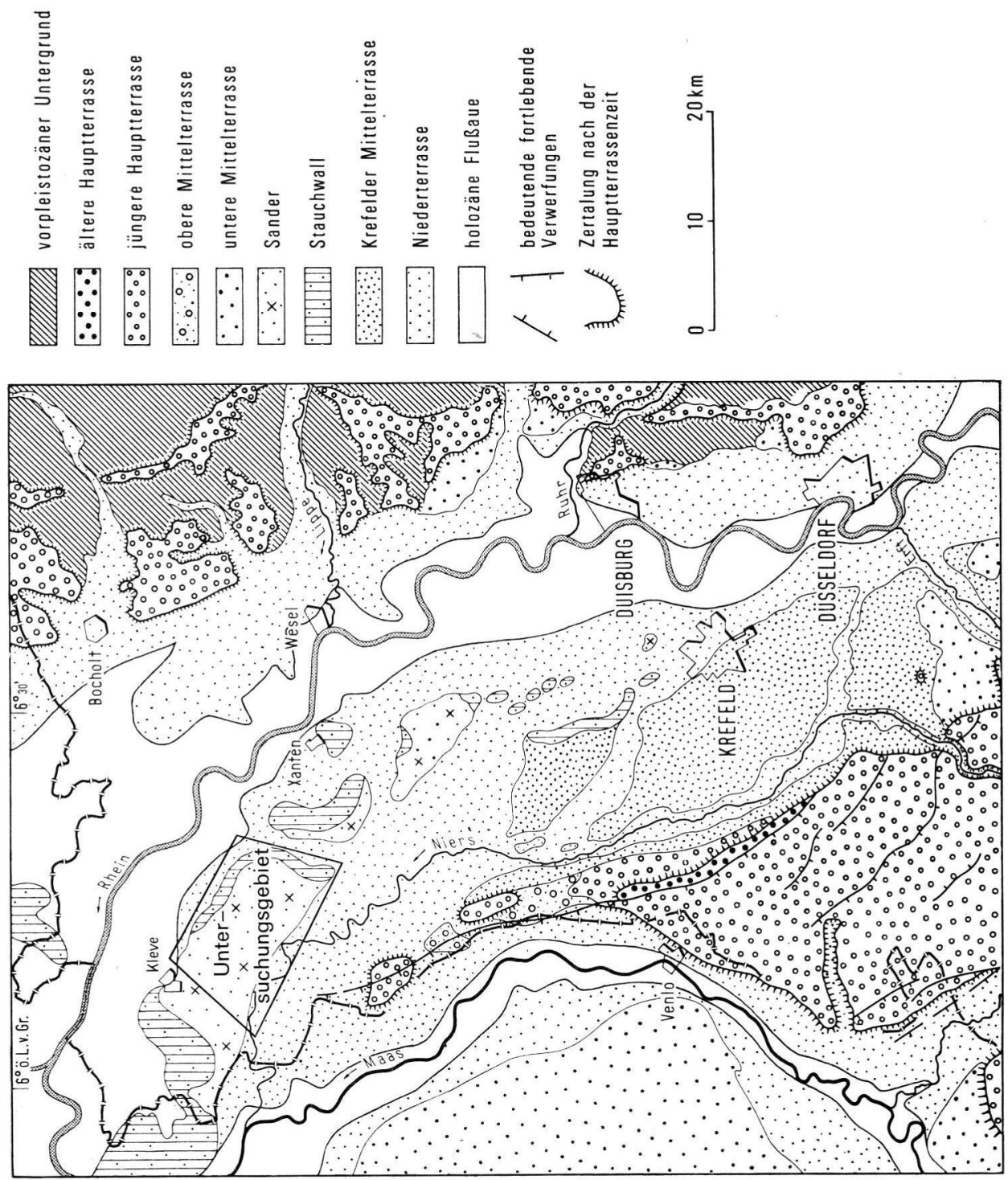

Abb. 1: Geologisch-geomorphologische Ubersichtskarte vom Niederrhein mit Untersuchungsgebiet (nach Braun 1968). 
Hier bieten sich Kennzahlen an, die durch ihren Zahlenwert die Fein heit oder Grobheit einer Sedimentprobe ausdrücken. Kennzahlen können miteinander in Beziehung gesetzt werden zu einer Raumstruktur und zum quantitativen Vergleich von errechneten Werten untereinander (SIEBERTZ 1980).

Kennzahlen sind in der Geowissenschaft zu den verschiedensten Zwecken benutzt worden. Nach LeSER (1977) dienen sie der Herausarbeitung von Charakteristika und Signifikanzen, wobei ihnen zum Vergleich von Reihenuntersuchungen eine wichtige Bedeutung zukommt (vgl. z. B. Hesemann-Kennzahl in Köster 1964 u. Leser 1977).

\section{Die Methode}

Schönhals (1955) hat den Feinheitsgrad als Kennzahl in die Literatur eingeführt. Der Terminus Feinheitsgrad bezieht sich auf die Fein he it bzw. Grobheit einer Sedimentprobe.

Für quartärgeomorphologische Fragen, die sich häufig mit dem Substrat des oberflächennahen Untergrundes befassen, ist diese sedimentologische Auswertungsmethode von großer Bedeutung $\left.{ }^{1}\right)$. Siebertz (1980) hat diese Methode für weichselzeitliche äolische Decksedimentuntersuchungen am unteren Niederrhein (Abb. 1) benutzt.

Die Errechnung des Feinheitsgrades ist recht einfach. Aus den Tabellen $1-3$ sind die einzelnen Schritte zu ersehen, die für eine sinnvolle mathematische und graphische Auswertung von Sedimentproben von Bedeutung sind. An folgendem Beispiel sei die Errechnung des Feinheitsgrades (FG) erläutert: der Summenkurve werden die Prozentwerte entnommen, wobei die nächst höhere Fraktion der vorhergehenden additiv aufgeschlagen wird. Mit der feinsten Kornfraktion wird begonnen. Die Endsumme (645,3 in Tab. 1) wird durch die Anzahl der Kornfraktionen (9) dividiert. Das Ergebnis ist der Feinheits$\left.\operatorname{grad} 71,7(645,3: 9)^{2}\right)$.

Tab. 1: Summenkurve und Feinheitsgrad einer Lößprobe vom unteren Niederrhein (A $30 \& \mathrm{r} 25.16980 ; \mathrm{h} 57.29840$ )

\begin{tabular}{|c|c|c|c|c|}
\hline \multicolumn{2}{|c|}{$\begin{array}{l}\text { Kornfraktionen } \\
\text { DIN } 4188\end{array}$} & \multirow{2}{*}{$\begin{array}{c}\begin{array}{c}\text { Anteile } \\
\text { in } \%\end{array} \\
0,1\end{array}$} & \multirow{2}{*}{$\begin{array}{c}\begin{array}{c}\text { Summenkurve } \\
\text { in } \%\end{array} \\
100,0\end{array}$} & \multirow{2}{*}{$\begin{array}{c}\text { Feinheitsgrad } \\
\text { FG }\end{array}$} \\
\hline $2-1$ & $\mathrm{~mm}$ & & & \\
\hline $1-0,63$ & ” & 0,4 & 99,9 & 545,3 \\
\hline $0,63-0,2$ & $"$ & 3,7 & 99,5 & 445,4 \\
\hline $0,2-0,1$ & $"$ & 1,7 & 95,8 & 345,9 \\
\hline $0,1-0,063$ & $"$ & 2,0 & 94,1 & 250,1 \\
\hline $0,06-0,02$ & $"$ & 61,1 & 92,1 & 156,0 \\
\hline $0,02-0,006$ & $"$ & 14,1 & $a(3) \quad 31,0$ & 63,9 \\
\hline \multirow{3}{*}{$<0,002$} & $"$ & 0,9 & $a(2) \quad 16,9$ & 32,9 \\
\hline & $"$ & 16,0 & $a(1) \quad 16,0$ & 16,0 \\
\hline & & 100,0 & & 71,7 \\
\hline
\end{tabular}

1) Bei den Kennzahlen handelt es sich nicht um absolute Werte, sondern um Vergleichszahlen für Substrate mit einer ähnlichen Korngrößenzusammensetzung. Diese Voraussetzung wird von dem Löß in idealer Weise erfüllt (SchöNHALS 1955). Untersuchungen an weichselzeitlichen äolischen Decksedimenten haben gezeigt, daß diese Methode an Sedimenten mit anderer Zusammensetzung ebenso gut anwendbar ist.

2) Die Addition der Kornfraktionsanteile ergibt die Summenkurve (Summe 100\%); die der Summenkurve die Endsumme zur Errechnung des Feinheitsgrades (Summe 645,3 in Tab. 1). 
Tab. 2: Summenkurve und Feinheitsgrad einer Sandlößprobe vom unteren Niederrhein (A 2 y r25.13360; h57.30680)

\begin{tabular}{|c|c|c|c|c|}
\hline \multicolumn{2}{|c|}{$\begin{array}{l}\text { Kornfraktionen } \\
\text { DIN } 4188\end{array}$} & \multirow{2}{*}{$\begin{array}{c}\text { Anteile } \\
\text { in \% } \\
-\end{array}$} & \multirow{2}{*}{$\begin{array}{c}\text { Summenkurve } \\
\text { in } \%\end{array}$} & \multirow{2}{*}{$\begin{array}{c}\text { Feinheitsgrad } \\
\text { FG } \\
599,6\end{array}$} \\
\hline $2-1$ & $\mathrm{~mm}$ & & & \\
\hline $1-0,63$ & ” & 0,4 & 100,0 & 499,6 \\
\hline $0,63-0,2$ & $"$ & 11,9 & 99,6 & 399,6 \\
\hline $0,2-0,1$ & $"$ & 2,6 & 87,7 & 300,0 \\
\hline $0,1-0,063$ & $"$ & 2,7 & 85,1 & 212,3 \\
\hline $0,06-0,02$ & $"$ & 59,4 & 82,4 & 127,2 \\
\hline $0,02-0,006$ & $"$ & 10,1 & 23,0 & 44,8 \\
\hline $0,006-0,002$ & ” & 4,0 & 12,9 & 21,8 \\
\hline \multirow[t]{2}{*}{$<0,002$} & 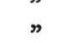 & 8,9 & 8,9 & 8,9 \\
\hline & & 100,0 & & 66,6 \\
\hline
\end{tabular}

Tab. 3: Summenkurven und Feinheitsgrade zweier Flugsandproben vom unteren Niederrhein (a: A $8 \gamma$ r25.21640; h57.28480 - b: A $17 \alpha$ r25.20180; h57.32680)

\begin{tabular}{|c|c|c|c|c|c|c|c|}
\hline \multirow{2}{*}{\multicolumn{2}{|c|}{$\begin{array}{l}\text { Kornfraktionen } \\
\text { DIN } 4188\end{array}$}} & \multicolumn{2}{|c|}{ Anteile in $\%$} & \multicolumn{2}{|c|}{ Summenkurven in $\%$} & \multicolumn{2}{|c|}{ Feinheitsgrade FG } \\
\hline & & a & $\mathrm{b}$ & $a$ & b & $a$ & b \\
\hline $2-1$ & $\mathrm{~mm}$ & 0,1 & 0,3 & 100,0 & 100,0 & 476,6 & 477,2 \\
\hline $1-0,63$ & 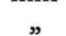 & 0,3 & 1,4 & 99,9 & 99,7 & 376,6 & 377,2 \\
\hline $0,63-0,2$ & ", & 22,6 & 30,5 & 99,6 & 98,3 & 276,7 & 277,5 \\
\hline $0,2-0,1$ & $"$ & 33,9 & 20,9 & 77,0 & 67,8 & 177,1 & 179,2 \\
\hline $0,1-0,063$ & $"$ & 7,6 & 10,0 & 43,1 & 46,9 & 100,1 & 111,4 \\
\hline $0,06-0,02$ & " & 24,2 & 23,9 & 35,5 & 36,9 & 57,0 & 64,5 \\
\hline $0,02-0,006$ & " & 5,6 & 4,8 & 11,3 & 13,0 & 21,5 & 27,6 \\
\hline $0,006-0,002$ & $"$ & 1,2 & 1,8 & 5,7 & 8,2 & 10,2 & 14,6 \\
\hline \multirow[t]{2}{*}{$<0,002$} & ” & 4,5 & 6,4 & 4,5 & 6,4 & 4,5 & 6,4 \\
\hline & & 100,0 & 100,0 & & & 53,0 & 53,0 \\
\hline
\end{tabular}

Die mathematische Formel zur Errechnung des Feinheitsgrades (FG) kann daher wie folgt definiert werden:

$$
\mathrm{FG}=\begin{gathered}
\mathrm{n} \\
\mathrm{i}=1 \\
\mathrm{i}
\end{gathered}
$$

Endsumme des Feinheitsgrades (z. B. 645,3) bildet und $\mathbf{n}$ im Nenner gleich neun $(9=$ Anzahl der Kornfraktionen) zu setzen ist.

\section{Praktische Anwendung}

Die Korngrößenzusammensetzung spielt bei der Untersuchung von (äolischen) Sedimenten eine wesentliche Rolle. Aus der Anderung der Körnung oder der Feinheit einer Probe können wichtige Schlüsse auf die Entstehung des Sediments gezogen werden. Kennzahlen dienen hier als geeignetes Mittel dazu, die Änderung der Feinheit verschiedener Proben quantitativ zu erfassen und diese in Abhängigkeit von anderen Faktoren graphisch darzustellen (ScHÖNHALs 1955).

Am unteren Niederrhein bedecken weichselzeitliche äolische Sedimente die saalezeitlichen Sanderflächen (Abb. 1). Diese Decksedimente wurden in der Vergangenheit verschiedentlich als Flugsande, Sandlösse und Flottsande/Flottlehme klassifiziert (BRAUn 1978; 
MüLler 1954, 1959), weil sie nördlich der klassischen Lößgrenze liegen, die entlang dem Mittelgebirgsrand verläuft und im Niederrheinischen Tiefland etwa die Linie KrefeldMönchen-Gladbach-Aachen beschreibt (vgl. Abb. 21 in Siebertz 1980). Isolierte Einzelvorkommen nördlich dieser Lößgrenze wurden meist als Sandlösse oder Flottsande angesprochen und chronologisch häufig mit den Flugsandablagerungen im nordwestdeutschen Raum gleichgesetzt (vgl. Müller 1959; SChraps u. a. 1978).

Im Rahmen der Erstellung des geomorphologischen Kartenblattes Kalkar im Maßstab $1: 25000$ (DFG-Forschungsprojekt Geomorphologische Detailkartierung in der Bundesrepublik Deutschland) erwuchs aus der Decksedimentkartierung das Problem der Lößsedimentation und Paläogeographie auf dem Niederrheinischen Höhenzug (Abb. 1). Die bislang unzureichenden Kenntnisse über die Decksedimente in diesem Raum führten zu Fragen nach der Herkunft und Verteilung der Sedimente, nach den Windverhältnissen zur Zeit der Ablagerung sowie nach der stratigraphischen Einordnung der Decksedimente in die Nomenklatur der fossilen Löß- und Bodenbildungen am Niederrhein (vgl. SieberTZ 1980).

Die Untersuchungsergebnisse, die mit Hilfe der Feinheitsgradmethode gewonnen wurden, sollen nun im einzelnen dargestellt werden:

(1). Bei der Fülle der vorhandenen Proben (vgl. Tab. 1 u. 2 in Siebertz 1980) bot sich der Feinheitsgrad zur Gliederung der unterschiedlich zusammengesetzten Sedimente als sehr praxisnah und flexibel. Tab. 4 zeigt die erarbeitete Nomenklatur, die der Decksedimentgliederung auf dem Niederrheinischen Höhenzug zugrundegelegt wurde (Abb. 3). Die errechneten Durchschnittswerte entstammen der granulometrischen Laboranalyse und bilden mittlere Repräsentativ- und Toleranzwerte zwischen den verschiedenen Sedimenten, wobei neben dem Feinheitsgrad charakteristische Kornfraktionen (Grobschluff und Mittelsand) als Hilfe zur feineren Gliederung herangezogen wurden. Bei den Flugsanden traten je nach Feinheit der Probe eine Verschiebung des Korngrößenmaximums vom Mittelsand $(0,63-0,2 \mathrm{~mm})$ zugunsten der gröberen Feinsandfraktion $(0,2-0,1 \mathrm{~mm})$ auf, so daß diese beiden Kornfraktionen zusammengezogen wurden $(0,63-0,1 \mathrm{~mm})$.

Um die Sedimente sinnvoll gliedern zu können und dabei nicht zu große Toleranzwerte in der Zusammensetzung benutzen zu müssen, wurde innerhalb der lößverwandten Sedimente zwischen einem Sandlöß und einem Flottsand unterschieden (s. Tab. 4). Der Flottsand gehört zu den lößverwandten Sedimenten und nimmt eine Zwischenstellung zwischen den Sandlössen und den fein sortierten Flugsanden ein.

(2). BraUn (1968) hat das Kartenblatt C 4302 Bocholt geologisch kartiert, welches den Raum des nördlichen Niederrheinischen Höhenzuges abdeckt. Die kartierten Decksedimente sind in einer vereinfachten Karte bei Klostermann (1980) dargestellt (Abb. 2). Ferner liegt eine pedologische Karte Blatt Bocholt 1:100 000 vor, deren bodenkundliche Klassifizierungen sich weitgehend mit der Sedimentverteilung in Abb. 2 decken, so daß beim Vergleichen beider Kartenwerke vielfach Übereinstimmungen zwischen Substrat und Boden vorhanden sind.

Mit Hilfe der Decksedimentnomenklatur hat Siebertz (1980) eine neue Verteilung der Sedimente auf dem Niederrheinischen Höhenzug vorgenommen (Abb. 3). Beide Abbildungen ( 2 u. 3) zeigen eine unterschiedliche räumliche Verteilung der Decksedimente, die ferner durch eine Gliederung der Sedimente in Flugsande und lößähnliche Ablagerungen ergänzt wird. Damit werden klar zwei unterschiedliche Sedimentationsräume herausgestellt: 1. Flugsandablagerungen im E und SE, 2. Löß-, Sandlöß- und Flottsandablagerungen im SW und im Zentrum der Sanderhochfläche.

Entsprechend der Decksedimentneuverteilung zeigen jüngere bodenkundliche Klassifizierungen für diesen Raum bei HeIDE (1978), daß zwischen der Verbreitung der Sedimente 


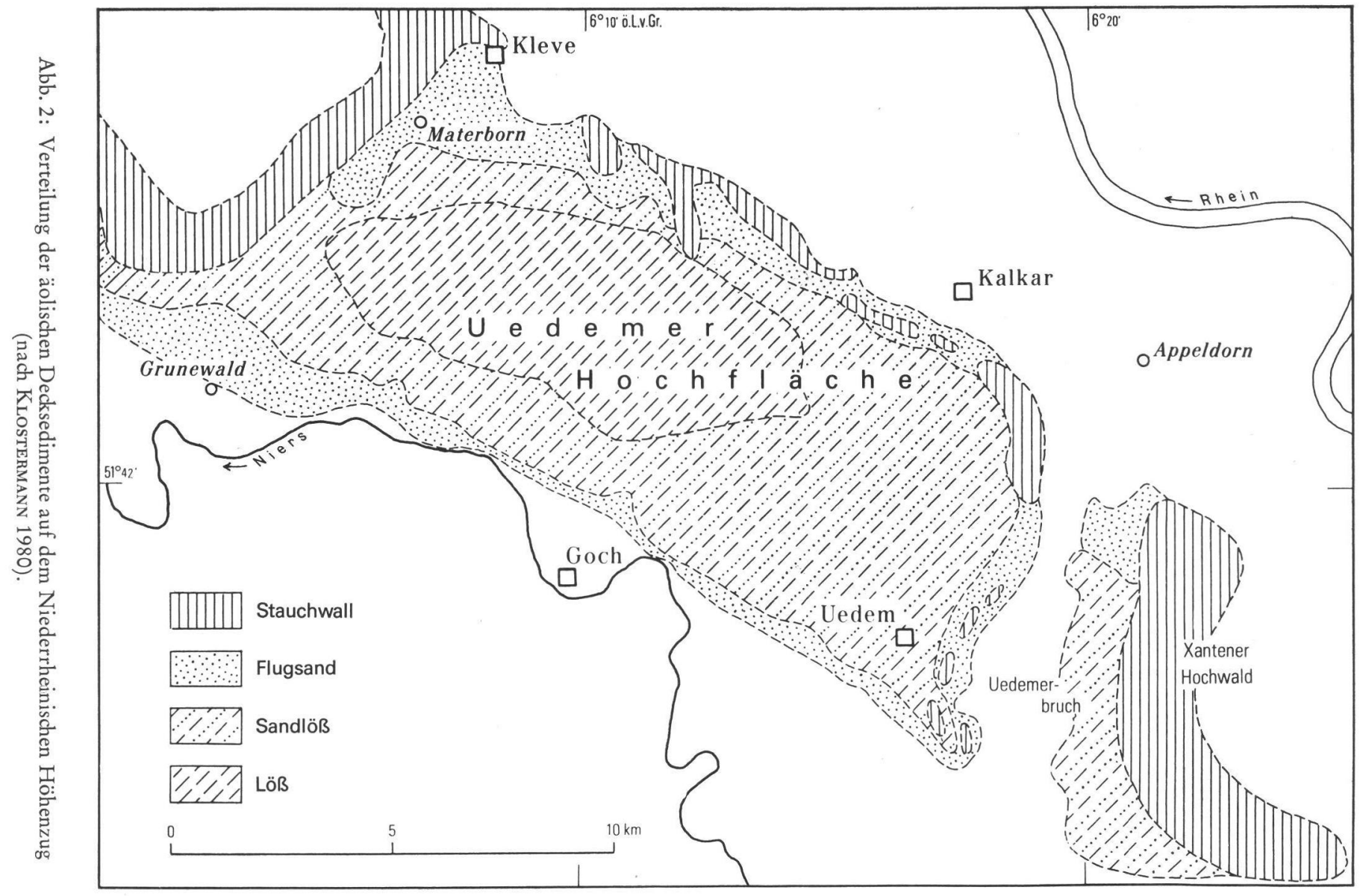




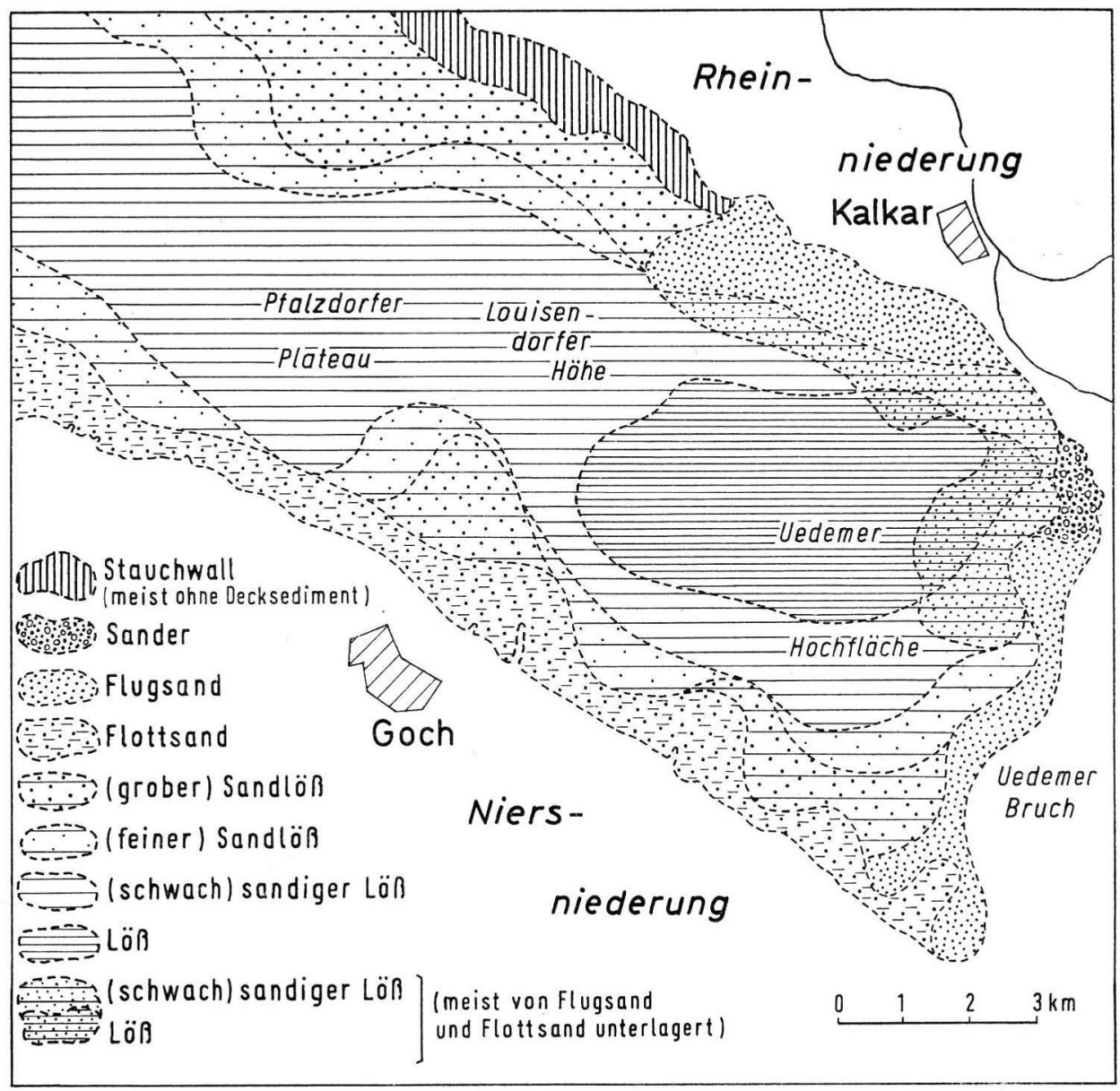

Abb. 3: Verteilung der äolischen Decksedimente auf dem Niederrheinischen Höhenzug (nach Siebertz 1980).

in Abb. 3 und den dazugehörigen charakteristischen Böden eine weitgehende Übereinstimmung vorliegt. Die Lösse bilden Parabraunerden, die (schwach) sandigen Lösse und Sandlösse Braunerden, die Flott- und Flugsande Podsol-Braunerden aus (s. HeIDE 1978).

(3). Schönhals (1955) bemerkt, daß durch eine Vermehrung der Beobachtungsstellen und der Proben in den einzelnen Profilen es wohl auch möglich wäre, "L i n i e n gle i cher Feinheit " zu rekonstruieren, die dann ein noch besseres Bild von den regionalen Unterschieden der Körnung vermittelten. Eine paläogeographische Rekonstruktion der Windrichtungen anhand von weichselzeitlichen Decksedimenten auf dem Niederrheinischen Höhenzug hat Siebertz (1980) vorgenommen (Abb.4). Dies konnte mit Hilfe des Feinheitsgrades sinnvoll durchgeführt werden, bei der gleiche Kennzahlenwerte durch Linien miteinander verbunden wurden (Linien gleicher Feinheit $=$ Isokatharosen) $\left.{ }^{3}\right)^{3}$.

3) In der griechischen Sprache ist der Begriff Feinheit nicht bekannt, sondern der Terminus Reinheit (= Katharos), der in der Bedeutung der Feinheit gleichkommt. Deshalb wurde der Terminus Reinheit durch den Begriff Feinheit ersetzt und dem griechischen Katharos gleichgesetzt. 


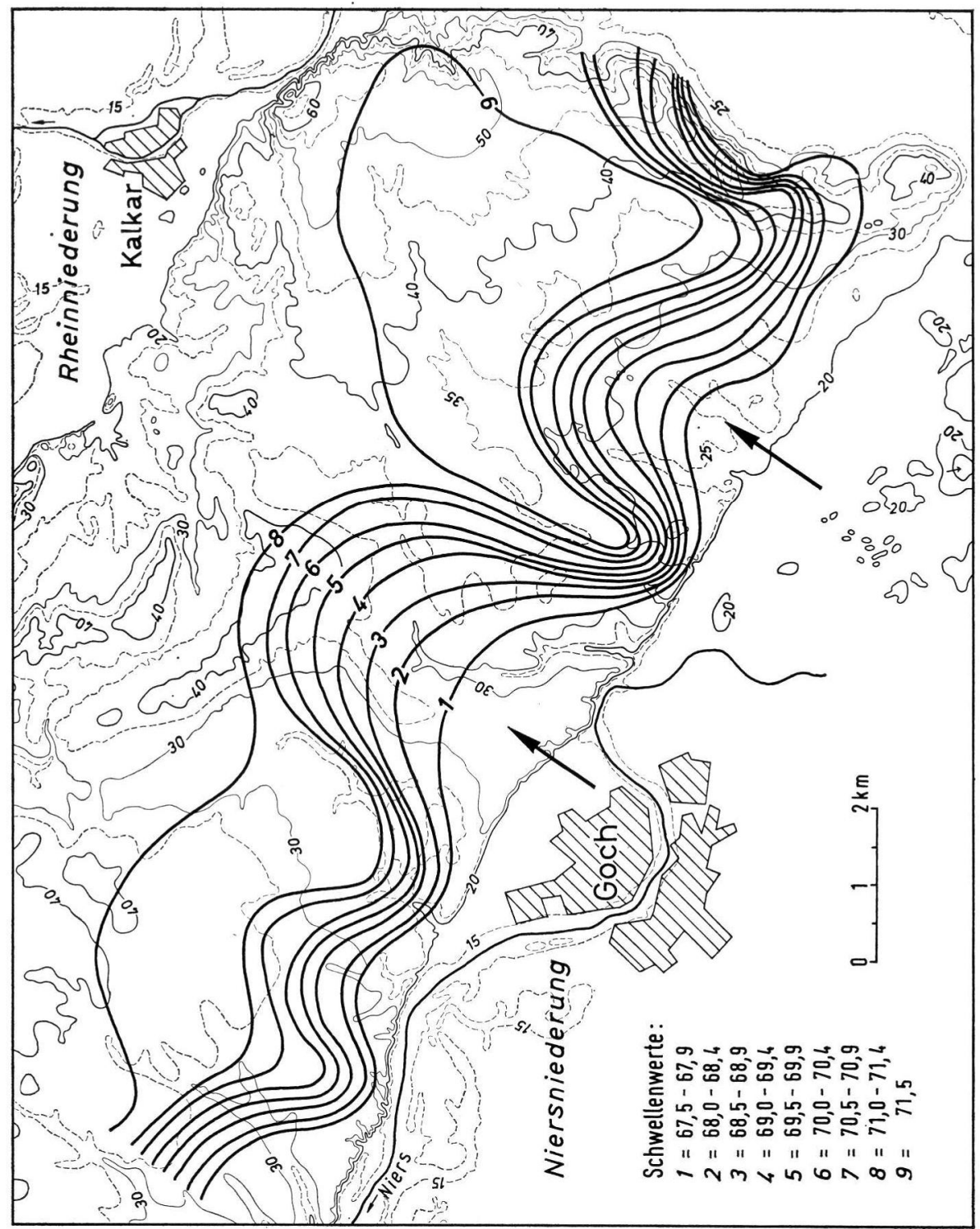

Abb. 4: Verteilung der Isokatharosen (Linien gleicher Feinheit) mit Windrichtungen.

Die Isolinien geben ein relatives Maß für die Windstärke, welche in der Aufeinanderfolge der Schwellenwerte zum Ausdruck kommt, die von SW zur Sanderhochfläche in der Feinheit zunehmen. Mit abnehmender Windgeschwindigkeit und Transportkraft kommt es zur Saigerung der feineren Sedimentbestandteile. Das Relief der Sanderhochfläche hat ferner Einfluß auf die Winddynamik und damit verbunden auf die Verbreitung der Sedimente. Aus dem Isolinienfeld in Abb. 4 läßt sich dies gut ableiten: flaches Relief im SW 
Tab. 4: Nomenklatur äolischer Decksedimente auf dem Niederrheinischen Höhenzug.

\begin{tabular}{|c|c|c|c|c|}
\hline Art & $\phi \mathrm{FG}$ & $\phi$ Grobschluff & $\begin{array}{c}\phi \text { Mittelsand } \\
(0,63-0,2 \mathrm{~mm})\end{array}$ & $\phi \begin{array}{c}\phi \text { Profilmächtig- } \\
\text { keiten }\end{array}$ \\
\hline $\begin{array}{l}\text { Flottsand } \\
\text { grober Sandlöß } \\
\text { feiner Sandlöß } \\
\text { (schwach) sandiger Löß } \\
\text { Löß }\end{array}$ & $\begin{array}{l}62-65 \\
67 / 68 \\
69 / 70 \\
71 \\
72\end{array}$ & $\begin{array}{l}49 \% \\
54 \% \\
57 \% \\
61 \% \\
62 \%\end{array}$ & $\begin{array}{c}15 \% \\
10-11 \% \\
7 \% \\
4-5 \% \\
2-3 \%\end{array}$ & $\begin{array}{r}60-80 \mathrm{~cm} \\
60-80 \mathrm{~cm} \\
60-80 \mathrm{~cm} \\
80-100 \mathrm{~cm} \\
100-120 \mathrm{~cm}\end{array}$ \\
\hline Art & $\phi \mathrm{FG}$ & \multicolumn{2}{|c|}{$\phi$ Grobschluff } & $\begin{array}{l}\text { Sandfraktionen } \\
, 63-0,1 \mathrm{~mm})\end{array}$ \\
\hline $\begin{array}{l}\text { grober Flugsand } \\
\text { feiner Flugsand }\end{array}$ & $\begin{array}{l}54 \\
59\end{array}$ & \multicolumn{2}{|c|}{$\begin{array}{l}23 \% \\
35 \%\end{array}$} & $\begin{array}{l}55 \% \\
40 \%\end{array}$ \\
\hline
\end{tabular}

bei Uedem, schnelles Saigern der Sedimente auf kurzem Raum und Ablagerung der feinsten Sedimente auf einer relativ großen Fläche (Isolinie 9. Schwellenwert), die weit an den südwestlichen Sanderhang (Niersniederung) heranreicht; nordwestlich davon ist eine Windgasse ausgebildet, deren feinste Isolinie (8. Schwellenwert) weit auf der Sanderhochfläche verläuft und im Abstand der Isolinien zueinander erkennen läßt, daß eine allmähliche Saigerung der Sedimente stattgefunden hat. Der Wind hat senkrecht zu den Isolinien geweht (Abb. 4).

Tab. 5: Sedimentologischer Aufbau eines Lößprofils vom unteren Niederrhein (r25.16980; h57.29840)

\begin{tabular}{ccc}
\hline $\begin{array}{c}\mathbf{A} \text { 30 } \\
\text { Tiefe in cm }\end{array}$ & Abschnitte & Feinheitsgrade FG \\
\hline & & \\
$20 \mathrm{~cm}$ & $\zeta$ & 70,0 \\
$40 \mathrm{~cm}$ & $\varepsilon$ & 71,7 \\
$60 \mathrm{~cm}$ & $\delta$ & 74,9 \\
$80 \mathrm{~cm}$ & $\gamma$ & 73,8 \\
$100 \mathrm{~cm}$ & $\beta$ & 72,4 \\
$120 \mathrm{~cm}$ & $\alpha$ & 70,1 \\
$140 \mathrm{~cm}$ & & $\overline{\mathrm{x}}=72,2$
\end{tabular}

Bei der Errechnung der Schwellenwerte wurde methodisch wie folgt vorgegangen (vgl. Tab. 5): für jeden Profilabschnitt $(20 \mathrm{~cm})$ wurde der Feinheitsgrad errechnet. Da gleiche Profilabschnitte verschiedener Aufschlüsse nicht syngenetisch abgelagert sein müssen, durften gleiche Kennzahlen dieser Profilabschnitte nicht unmittelbar miteinander in Beziehung gesetzt werden. Nach der Annahme, daß unter gleichbleibender Windstärke zur gleichen Zeit gleichmäßige äolische Ablagerungen gebildet wurden, erfolgte für homogen aufgebaute Profile die Errechnung einer Mittelwertskennzahl (Beispiel A $30=\bar{x}$ FG 72,2 in Tab. 5), die für alle an diesem Profil beteiligten Abschnitte als repräsentativ angesehen wurde. Profile mit gleichen Mittelwertskennzahlen wurden durch eine Linie miteinander verbunden (Isokatharosen); Profile mit meist inhomogenem Sedimentaufbau erhielten keine Mittelwertskennzahl (Abb. 4). Dies kann von verschiedenen Faktoren abhängig sein: keine regelmäßige Sedimentation, geringmächtige Profile, Verunreinigungen im Profil durch Umlagerungen. 


\section{Probleme in der Anwendung}

Für die Quartärforschung, die u. a. verstärkt auf der Basis von Korngrößenanalysen arbeitet und von Geologen, Pedologen sowie Geomorphologen gleichermaßen angesprochen wird, ist der Feinheitsgrad eine wichtige Stütze und Hilfe. Er ist ein relatives Maß für die günstigste Konstellation der an einer Probenzusammensetzung beteiligten Kornfraktionen. Je höher die Kennzahl, desto feiner ist die Probe; je niedriger die Kennzahl, um so gröber ist sie (Schönhals 1952). Als Beispiel seien hier die Kennzahlen aus den Tab. 1-3 genannt, die deutlich die unterschiedlichen Feinheitsgradstufen der verschiedenen Sedimente zum Ausdruck bringen. $\mathrm{Zu}$ beachten ist, daß sich der Feinheitsgrad entsprechend dem Devisor ändert, der aus der Anzahl der Kornfraktionen gebildet wird, so daß sich bei $\mathrm{Zu}$ - oder Abnahme der Kornfraktionsanzahl der Genauigkeitsgrad des Feinheitsgrades (leicht) ändert.

Bei der Bearbeitung der äolischen Decksedimentproben vom Niederrheinischen Höhenzug hat sich gezeigt, daß ein hoher Feinheitsgrad innerhalb eines Vertikalprofils (z. B. Lößprofil A 30 in Tab. 5) nicht unbedingt mit einem $B_{t}$-Horizont im pedologischen Sinne zusammenfallen muß. Je nach Zusammensetzung der Probe kann der erhöhte Tongehalt einen Einfluß auf den Feinheitsgrad ausüben, jedoch zeigte sich beim Errechnen der Kennzahl, daß (meist) der hohe Grobschluffanteil bei den Lössen die Feinheit der Kennzahl steuert (Siebertz 1980). Tonanreicherungsbänder können mit dieser Methode wohl lokalisiert werden, Unterschiede zwischen Verlehmungen und Lessivierungen müssen allerdings im Labor mit speziellen Untersuchungsmethoden erarbeitet werden.

Die Praxis zeigte, daß sich die Kennzahlen für Nomenklatur- und Gliederungszwecke sehr gut eignen. Hier ist allerdings zu beachten, daß neben dem Feinheitsgrad noch andere Werte zu Rate gezogen werden sollten, z. B. charakteristische Kornfraktionen, die für den sedimentologischen Aufbau einer Probe von Bedeutung sind. Es sind die Kornfraktionen des Grobschluffs und des Mittelsandes (0,63-0,2 mm) bzw. bei Flugsanden häufig im Wechsel die Fraktionen des gröberen Feinsandes $(0,2-0,1 \mathrm{~mm})$ mit der des Mittelsandes oder der Mittelwert beider Fraktionen zusammen $(0,63-0,1 \mathrm{~mm})$.

\section{Zusammenfassung}

Für die praktische Anwendung der Feinheitsgradmethode in der Quartärmorphologie bieten sich zusammenfassend folgende Möglichkeiten an:

1) Gliederung unterschiedlicher Sedimentationsräume (bei einer flächendeckenden sedimentologischen Bearbeitung eines geographischen Raumes)

2) Vergleich von (unterschiedlichen) Proben und Sedimentationsräumen miteinander (Voraussetzung ist hier die gleiche methodische Bearbeitung und Auswertung der Proben)

3) Erstellung von sedimentologischen Klassifikationen und Nomenklaturen (für die Bildung von Schwellenwerten sollten neben der Kennzahl charakteristische Korngruppen herangezogen werden)

4) Lokalisierung von starken Verlehmungsvorgängen und Tonanreicherungen innerhalb eines Sedimentprofils (die Ursachen - Verwitterung oder Lessivierung werden damit nicht geklärt)

5) Rekonstruktion von Windrichtungen zur Zeit der Sedimentablagerungen und damit verbunden Rückschlüsse auf die Paläogeographie

6) Bestimmung und Ableitung der Bodenart. 


\section{Schriftenverzeichnis}

Braun, F. J. (1968): Ubersichtskarte von Nordrhein-Westfalen $1: 100000$, Erläuterungen zur geologischen Karte C 4302 Bocholt, 13-92, 12 Abb., 4 Tab., 4 Taf.; Krefeld.

- (1978): Zur Herkunft und Zusammensetzung des "Sandlösses" auf der Uedemer SanderHochfläche (Niederrhein). - Fortschr. Geol. Rheinld. u. Westf., 28: 335-343, 5 Abb., 3 Tab.; Krefeld.

Heide, G. (1978): Boden und Bodennutzung. - In: Geologie am Niederrhein, 35-38, 1 Abb.; Krefeld (Geol. L.-Amt Nordrhein-Westfalen).

Klostermann, J. (1980): Die Ursachen der konzentrischen Anordnung von Flugsand, Sandlöß und Löß auf der Uedemer Hochfläche. - Der Niederrhein, 47: 1-5, 4 Abb.; Krefeld.

Köster, E. (1964): Granulometrische und morphometrische Meßmethoden an Mineralkörnern, Steinen und sonstigen Stoffen. - 336 S., 109 Abb., 68 Tab., Stuttgart (Enke).

Leser, H. (1966): Pecolologisch-sedimentologische Untersuchungen als geomorphologische Methode. - Forschungen u. Fortschritte, 40: 296-300; Berlin.

- (1977): Feld- und Labormethoden der Geomorphologie. — 446 S., 91 Abb., 28 Tab., Berlin (de Gruyter).

Müller, E. H. (1954): Über die Herkunft des Lösses im Rheinland und im südlichen Westfalen. Geol. Jb., 69: 401-406, 1 Taf., Hannover.

- (1959): Art und Herkunft des Lösses und Bodenbildung in den äolischen Ablagerungen Nordrhein-Westfalens unter Berücksichtigung der Nachbargebiete. - Fortschr. Geol. Rheinld. u. Westf., 4: 255-265, 1 Abb.; Krefeld.

Siebertz, H. (1980): Weichselzeitliche äolische Sedimente des Reichswaldes (unterer Niederrhein) und ihr paläogeographischer Aussagewert. - Diss. Univ. Bonn. 143 S., 23 Abb., 10 Tab.; Bonn.

SснӧNнALs, E. (1952): Gesetzmäßige Beziehungen zwischen Körnung und Kalkgehalt des Lösses und die Erkennung von Verwitterungszonen mit Hilfe der typischen Streubereiche. Geol. Jb., 66: 291-304, 7 Abb., 3 Tab.; Hannover.

- (1955): Kennzahlen für den Feinheitsgrad des Lösses. - Eiszeitalter u. Gegenwart, 6: 133147, 10 Abb., 8 Tab.; OOhringen.

Schraps, W. G. u. a. (1978): Zur Nomenklatur quartärer bodenbildender Substrate NordrheinWestfalens. - Fortschr. Geol. Rheinld. u. Westf., 28: 507-517, 1 Tab.; Krefeld.

Manuskript eingegangen am 8. 1. 1982. 
